\title{
NB-IoT and eMTC: Engineering results towards 5G/IoT Mobile Technologies
}

\author{
https://doi.org/10.3991/ijim.v13i01.9728
}

\author{
Ahmed El Mahjoubi ( $\left.{ }^{\varpi}\right)$, Tomader Mazri, Nabil Hmina \\ National School of Applied Sciences, Kenitra, Morocco \\ elmahjoubi.ahmed@univ-ibntofail.ac.ma
}

\begin{abstract}
G RF technology is an essential part of the wireless mobile technology. Technically, $5 \mathrm{G}$ is predicted to empower people-thing and thingthing interconnections by combining wireless technologies and networks. Our university has been actively support research on $4 \mathrm{G} / 5 \mathrm{G} / \mathrm{IoT}$, in which the NBIoT, eMTC and Massive Carrier Aggregation new features challenge are of big interest. New $5 \mathrm{G}$ trials put forward a lot of prerequisites for new RF interface in terms of radio bandwidth, power issues, as well as the huge number of connections for IoT capacity management and optimization. Based on Morocco's current situation, this paper first discusses the $5 \mathrm{G}$ technologies; In addition, we will discuss IoT SoftRadio application support, and NB-IoT master plan recap. At last, based on the current plan of $5 \mathrm{G}$ RF KPIs management according to first Morocco 5G trail Results and Deployment plans.
\end{abstract}

Keywords-NB-IoT, 5G, eMTC, eMIMO, CA

\section{Introduction}

$5 \mathrm{G}$ introduced a new radio technology for wireless. It is mainly used for scenarios that this feature provides: low rate, deep coverage, low power consumption, and considerable connections.

\section{Nb-IoT Radio and Performance}

Fig. 1 illustrates the mapping Application scenarios of IoT.

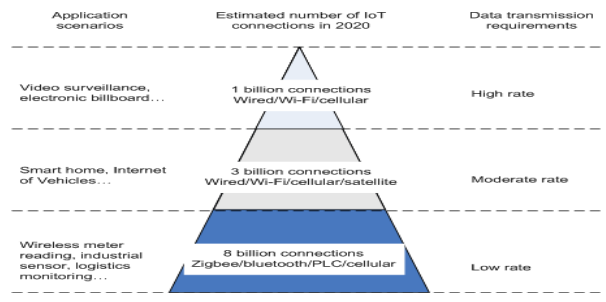

Fig. 1. Application scenarios of IoT 
Ref [2] introduce the basic concepts of NB-IoT include physical channel, physical channel frequency-domain structure, physical channel time-domain structure, coverage level, and aggregation level.

Physical Channel: In [3] there are three types of downlink physical channels for NBIoT, which are described as follows:

- Narrowband Physical Broadcast Channel $(\mathrm{NPBCH})$ : responsible for transmitting master information blocks (MIBs).

- NPDCCH: responsible for carrying the downlink control information (DCI).

- NPDCCH resources are allocated to UEs in units of control channel elements (CCEs).

- NPDSCH: responsible for carrying downlink data.

- There are two types of uplink physical channels for NB-IoT, which are described as follows:

- NPUSCH: responsible for carrying uplink data.

- NPRACH: responsible for carrying random access messages.

Fig. 2 illustrates the mapping relationships between physical channels and transport channels.

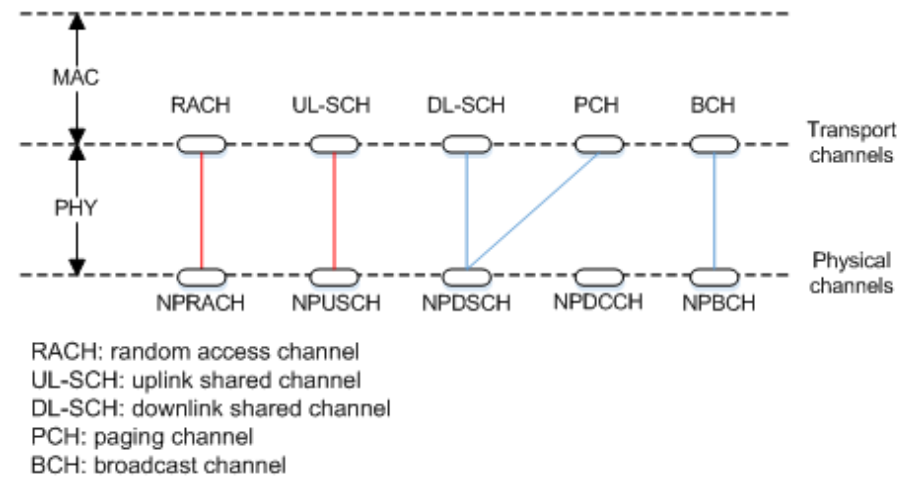

Fig. 2. Mapping relationships between physical channels and transport channels

NB-IoT RF Deployment Mode Proposal: This section describes the Standalone Deployment of NB-IoT; there are two options for standalone deployment:

Refarming: Refarming enables part of the spectrum resources for a RAT to be used by NB-IoT, without affecting the functionalities of that RAT. Typically, GSM spectrum resources are spared for NB-IoT by refarming with guard bands reserved between them. The GSM network is replanned to minimize the impact of refarming on GSM services. [3]

Figure 3-1 uses 1:1 deployment as an example. In this example, two GSM carriers are allocated to the NB-IoT network and a guard band of $100 \mathrm{kHz}$ is reserved between NB-IoT and GSM. In refarming deployment, the GSM frequencies in the entire buffer zone area need to be refarmed to reduce interference even if NB-IoT is not deployed. [2] 


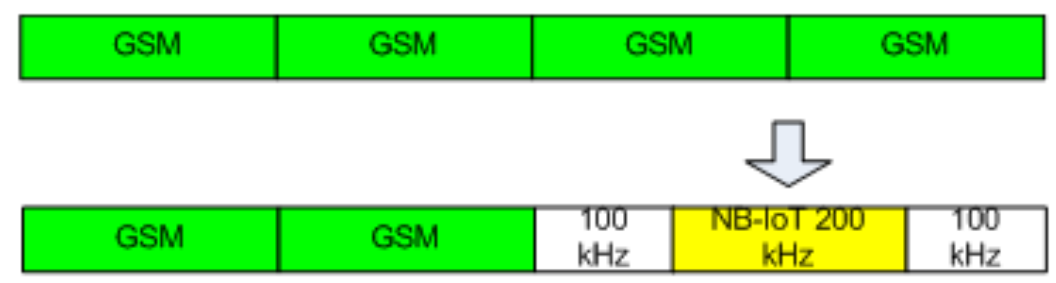

Fig. 3. Refarming deployment

Using Idle Spectrum Resources: Operators may own spectrum resources with non-standard bandwidths, which do not meet the communications requirements of certain RATs and therefore are not in use. Using these resources, NB-IoT can implement narrowband communications. Deploying NB-IoT on idle spectrum resources requires that sufficient guard bands be reserved between NB-IoT and those RATs, preventing impact on existing networks. The application scenarios are as follows: [3]

2 .

GSM idle spectrums are used to deploy the NB-IoT network, as shown in Figure 3-

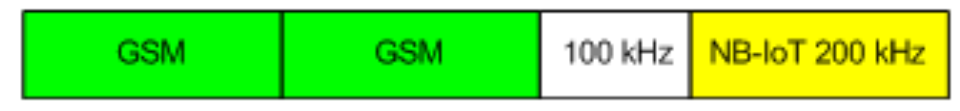

Fig. 4. NB-IoT deployment on GSM idle spectrum resources

UMTS idle spectrums are used to deploy the NB-IoT network, as shown in Figure 3-3. [2]

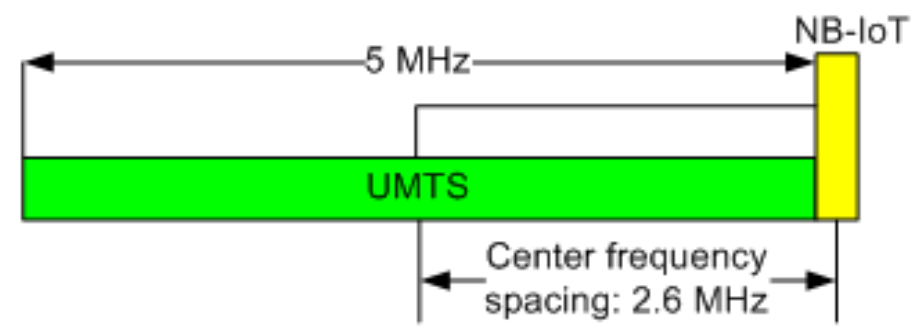

Fig. 5. NB-IoT deployment on UMTS idle spectrum resources

LTE idle spectrums are used to deploy the NB-IoT network, as shown in Figure 34. 


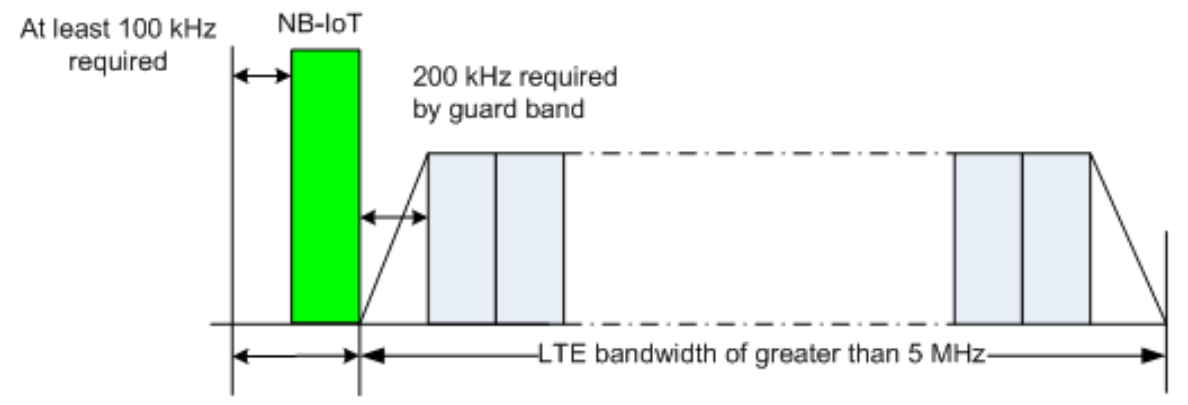

Fig. 6. NB-IoT deployment on LTE idle spectrum resources

This section describes the LTE Guardband Deployment of NB-IoT. [3]

To prevent adjacent-carrier interference or inter-RAT interference for existing RATs, a certain bandwidth must be reserved in addition to the valid bandwidth. This bandwidth is referred to as "guard band". The guard band between carriers is generally greater than or equal to $180 \mathrm{kHz}$. NB-IoT is a narrowband communication technology in which a bandwidth of $180 \mathrm{kHz}$ is allocated to both the uplink and downlink. Therefore, services can be deployed on the guard bands of existing RATs, which eliminates the need for new spectrum and improves the utilization of old spectrum.

In guard band deployment, NB-IoT services are now deployed on LTE FDD guard bands, as shown in Figure 3-5. This deployment mode must meet the requirements specified in 3GPP TS 36.101 (Release 13). [1]

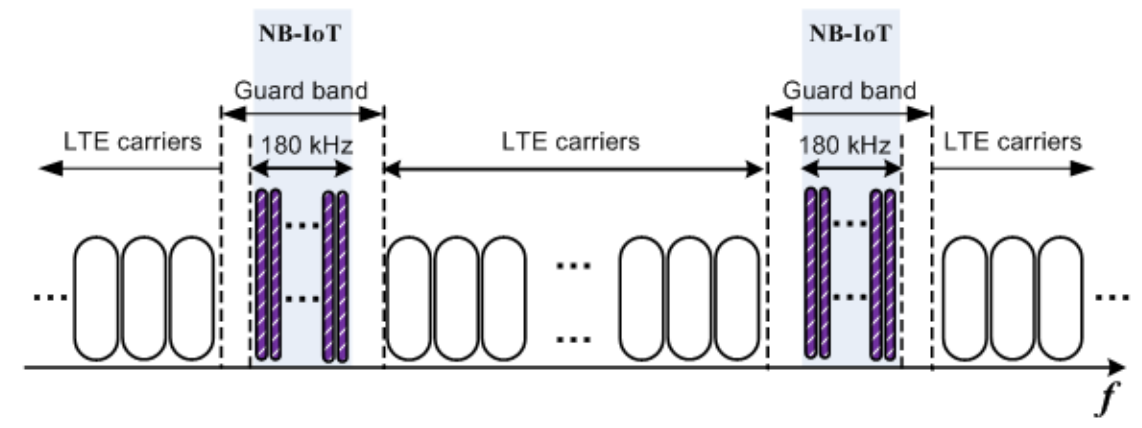

Fig. 7. LTE guard band deployment

For example, if the LTE FDD bandwidth is greater than or equal to $10 \mathrm{MHz}$, guard bands are sufficient for NB-IoT deployment, as shown in Figure 3-6. If the LTE FDD bandwidth is less than $10 \mathrm{MHz}$, the NB-IoT network cannot be deployed because of guard band insufficiency. [3] 


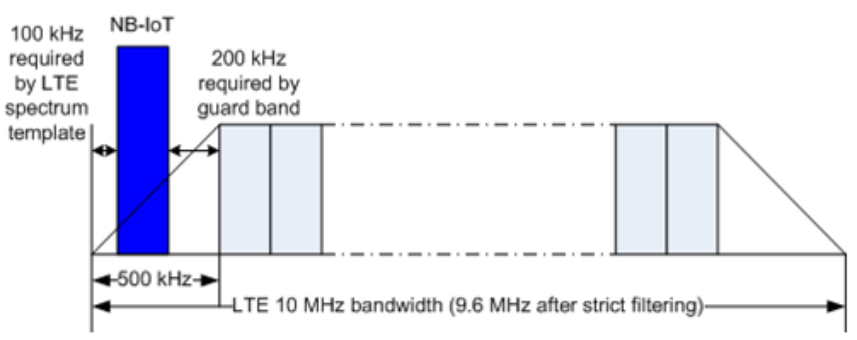

Fig. 8. LTE guard band deployment

(10 MHz LTE FDD bandwidth as an example)

In-band deployment is a typical deployment scenario, in which operators deploy NB-IoT using existing LTE FDD in-band RBs, as shown in Figure 3-7. [3]

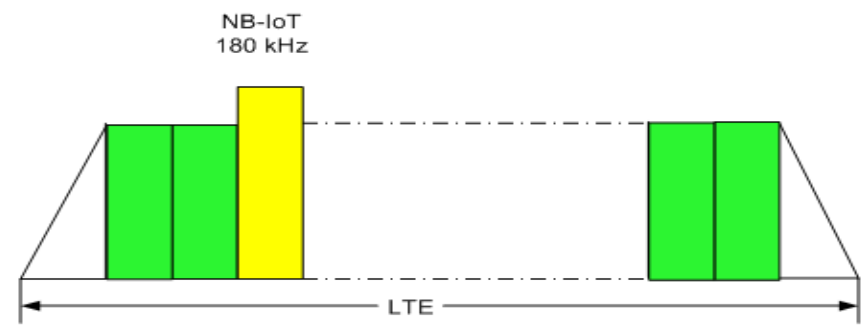

Fig. 9. LTE In-band deployment

\section{EMTC Radio and Network Performance}

eMTC is an IoT technology evolved on basis of the 3GPP protocols. It is mainly used for scenarios that feature medium and low rates, deep coverage, low power consumption, and massive connections.

eMTC provides the following benefits: [5]

- Maximum spectrum utilization

- eMTC can be deployed on existing LTE networks, fully utilizing current spectrum resources of operators for maximized spectral usage.

- Support for a large number of low- and medium-rate users

- The low-rate and low-activity service model supports a large number of users.

- Deep coverage

- The coverage gains provided by eMTC are $15 \mathrm{~dB}$ greater than those provided by a common LTE network with the help of time-domain repetition and other technologies.

- Low power consumption of UEs

- By using enhanced discontinuous reception (eDRX) and power saving mode (PSM), eMTC shortens the receive/transmit duration of UEs and therefore reduces their power consumption. 
Figure 4 illustrates the end-to-end network architecture of eMTC.

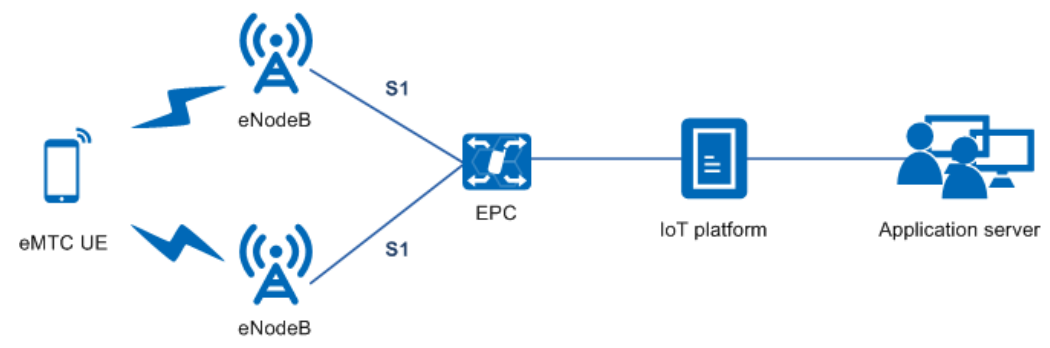

Fig. 10. eMTC network architecture

To ensure normal functioning of eMTC UEs on an 5G network, this feature performs enhanced processing on the following aspects: [2]

- Physical channel management

- Cell management

- Idle mode management

- Connected mode management

- Overload control

- DRX for UEs in connected mode

- Random access control and RACH optimization

- Scheduling

- Power control

- LCS

M2M services have high power saving requirements. This feature is introduced to save power of eMTC UEs and support the M2M solution with low power consumption to prolong UE standby time and improve user experience. [4]

By extending paging cycles for UEs in idle mode, this feature reduces their monitoring times and power consumption. [5]

eMTC UEs and common UEs coexist in a cell. The cell management procedures for eMTC UEs are the same as those for common UEs.

The bandwidth of an eMTC-capable cell must be $5 \mathrm{MHz}$ or higher. [5]

\section{Network Impact}

System Capacity

- Downlink traffic volume and throughput in the cell

- Downlink traffic volume and throughput will decrease in a cell serving both eMTC and common UEs. This is because they share PRB resources in the cell and MIB, SIB, and services for eMTC will consume PRB resources. Therefore, PRB resources available to common UEs decrease.

- Uplink traffic volume and throughput in the cell

- Uplink traffic volume and throughput will decrease in a cell serving both eMTC and common UEs. This is because they share PRB resources in the cell and 
PRACH, PUCCH, and eMTC services will consume PRB resources. Therefore, PRB resources available to common UEs decrease.

- CPU Usage

- CPU usage will increase, as a result of system processing for eMTC UEs.

- User-perceived throughput

UL/DL throughput perceived by users of common UEs will decrease in a cell serving both eMTC and common UEs because they share PRB resources in the cell. Therefore, PRB resources available to common UEs decrease. [4]

\section{Network Performance}

- The RRC setup success rate and handover rate may decrease in a cell serving both eMTC and common UEs because the total number of RRC connection resources in the cell is fixed. The RRC connection setup failure possibility increases, as the RRC connection specifications available to common UEs decrease. [3]

- When there are a large number of UEs in coverage enhancement mode, the number of messages that require retransmission will increase. The number of users that can access the cell will decrease as a result of SRI resource insufficiency.

- After eMTC is enabled, the online duration of common UEs will increase as a result of their reduced throughput. The average number of users will therefore slightly increase in the cell. The access of eMTC UEs to the cell will also contribute to the increase in the average number of users.

- PRB usage will increase in a cell serving both eMTC and common UEs. This is because they share PRB resources in the cell and eMTC UEs consume UL PRB overhead. Counters related to uplink MCS, BLER, and other factors are more likely to fluctuate because major changes will occur to the PRB resource usage and scheduling resource allocation will be more fragmented in an eMTC-enabled cell.

- PRB usage will increase in a cell serving both eMTC and common UEs. This is because they share PRB resources in the cell and eMTC UEs consume DL PRB overhead. After eMTC is enabled, PRB resource allocation for scheduling will be more fragmented and counters related to downlink MCS, BLER, CQI, and other factors are more likely to fluctuate. When RBG fragments cannot be fully used in a high load scenario, the DL PRB usage will slightly decrease and the number of DL scheduling UEs in each TTI may increase. This will increase the DL CCE usage.

With the fast evolution of radio communication technologies, radio communication is not limited only to radio calls. With the development of the Internet of Things (IoT), communications between machines are becoming very important. Our paper defines this type of communications as machine type communication (MTC).

\section{$4 \quad$ NB-IOT 5G Trial Results and Discussion}

The SoftRadio is a type of software used as a substitution of Narrowband Internet of Things (NB-IoT) eNodeBs and evolved packet cores (EPCs) during testing. 
The SoftRadio helps NB-IoT UE developers make and verify NB-IoT UEs, without deploying the NB-IoT eNodeB and EPC and using NB-IoT UE chip display teams, greatly improving NB-IoT UE commissioning efficiency.

Figure 5.1 illustrates the position of the SoftRadio on a network.

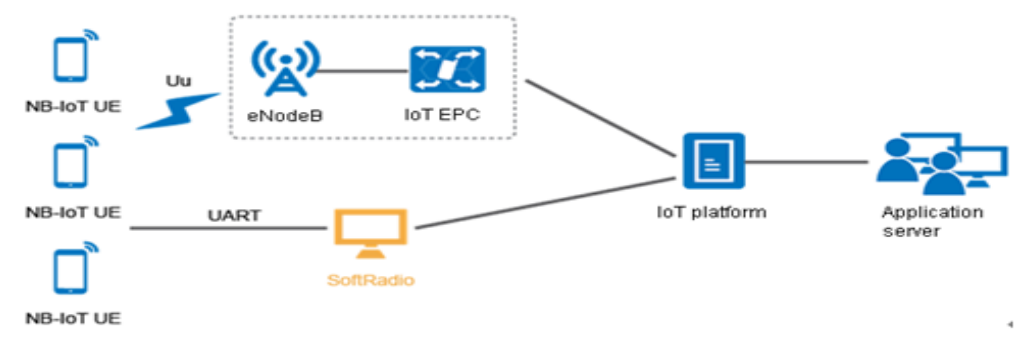

Fig. 11. Network architecture

- Functions of network elements (NEs) on the above network are as follows: [3

- NB-IoT UE: NB-IoT UEs, such as smart water meters and gas meters, are connected to an eNodeB over the Uu interface.

- SoftRadio: The SoftRadio is a type of software used to substitute NB-IoT eNodeBs and EPCs during testing.

- eNodeB: An eNodeB processes messages concerning network access over the Uu interface, manages cells, and forwards non-access stratum (NAS) data to a higherlayer NE. An eNodeB is connected to the NB-IoT EPC over the S1-lite interface.

- NB-IoT EPC: An NB-IoT EPC exchanges information with NB-IoT UEs at the NAS layer and forwards data related to NB-IoT services to the IoT platform.

- IoT platform: The IoT platform converges different types of IoT data from various access networks and then forwards the data to a required service application based on the data type.

- Application server: An application server works as an IoT data convergence point and processes data in compliance with customer requirements.

\section{Adding and Registering an NB-IoT Device}

- In the navigation tree of the SoftRadio interface, click Dashboard to navigate to the Dashboard interface.

- On the Dashboard interface, click next to Devices to expand the Add Device interface, as illustrated in Figure 5-2.

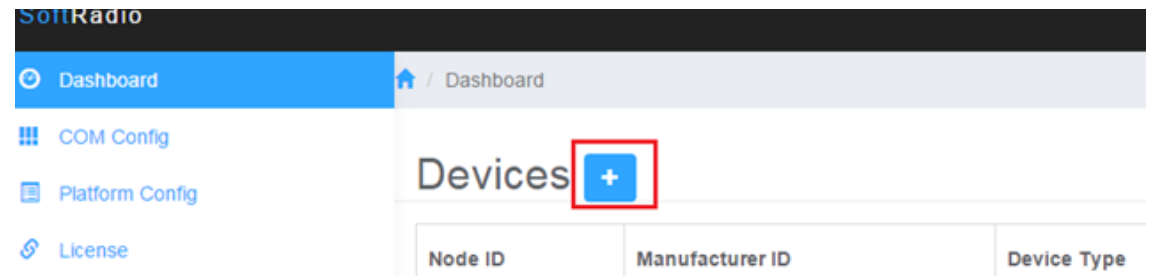

Fig. 12. Add Device interface 
In the Add Device interface, enter required device information under Device Info, as illustrated in Figure 5-3.

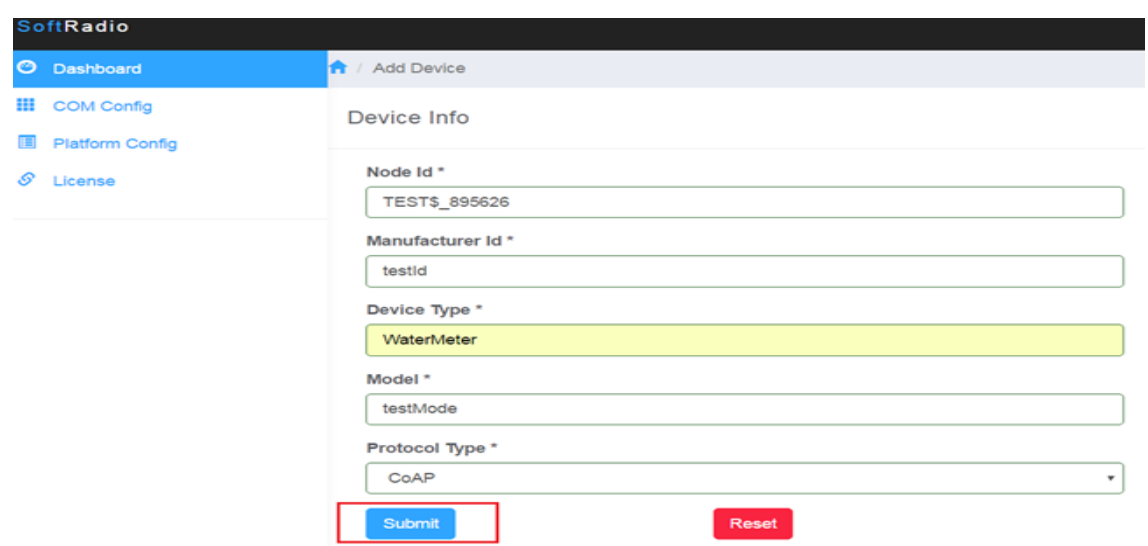

Fig. 13. Device Info interface

- Descriptions of required device information: Node Id uses the verification code obtained from the IoT platform, and must be entered in the format of TESTS_UUID. An NB-IoT UE manufacturer can obtain this verification code from the communication interface between an application and the IoT platform. [6]

- Manufacturer Id, Device Type, and Model must be consistent with information on the device profile created on the IoT platform, and such information is provided by the NB-IoT UE manufacturer. [6]

\section{Protocol Type uses CoAP by default.}

- Click Submit to submit device information. After such information is successfully submitted, information on the added NB-IoT device will be displayed on the Dashboard interface, and the device status will be displayed as Registered.

Figure 5-4 illustrates device info interface.

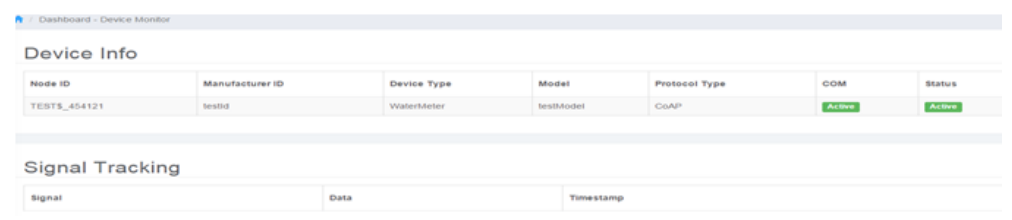

Fig. 14. Device Info interface

Information on the Signal Tracking page is the main basis for commissioning using the SoftRadio. During the commissioning, you can check AT commands sent to the SoftRadio from NB-IoT UEs, responses from the SoftRadio to NB-IoT UEs, and requests sent to the IoT platform from the SoftRadio on the Signal Tracking page. [7]

Table 1 Describe Signaling Tracking Types 
Table 1. Describing Signaling Tracking Types

\begin{tabular}{|l|l|}
\multicolumn{1}{c|}{ Type } & \multicolumn{1}{c|}{ Description } \\
\hline DEV->SR & AT commands sent to the SoftRadio from NB-IoT UEs \\
\hline SR->DEV & AT command responses sent to NB-IoT UEs from the SoftRadio \\
\hline SR->IOT & Requests sent to the IoT platform from the SoftRadio \\
\hline AT_ERROR & AT commands that cannot be identified by the SoftRadio \\
\hline
\end{tabular}

Typical MO/MT Procedure: Our trial of water meter is used as to introduce the $\mathrm{MO} / \mathrm{MT}$ procedure. Figure 5-5 illustrates MO/MT procedure. [8]

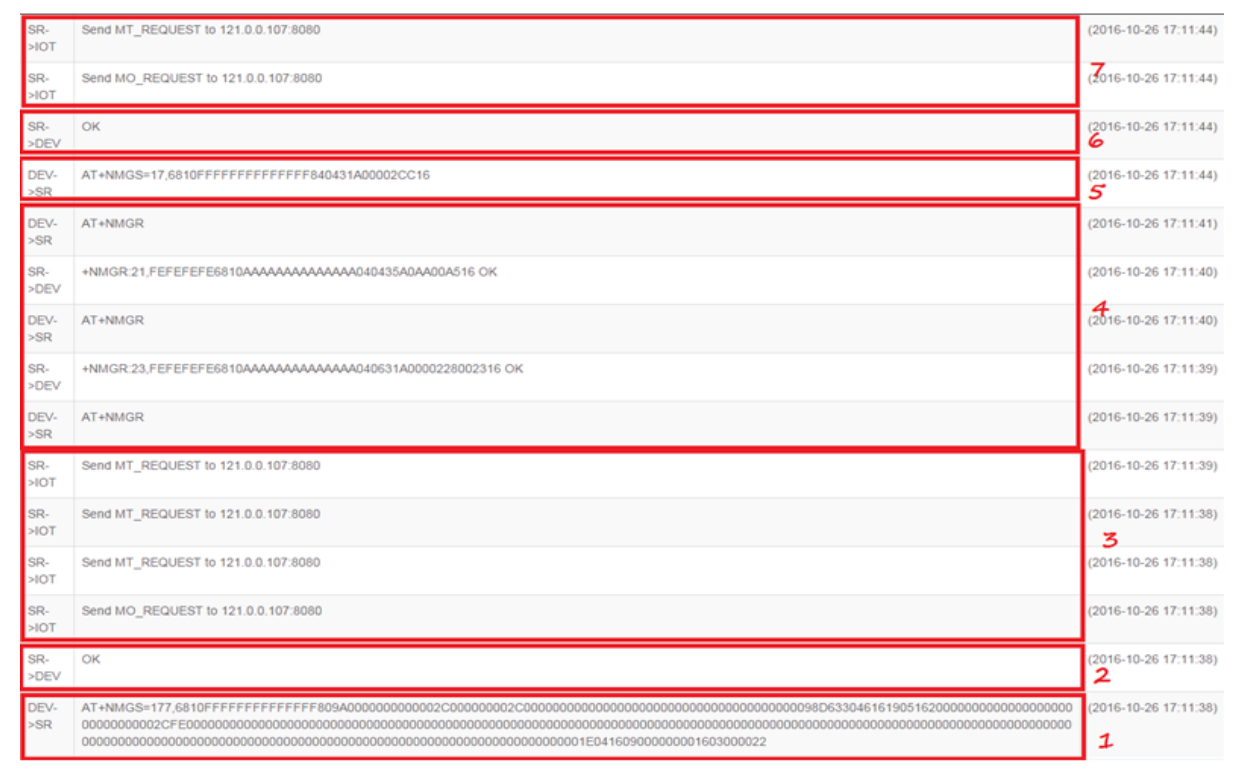

Fig. 15. MO/MT procedure

The MO/MT procedure is as follows:

- The water meter sends an AT+NMGS command to request for sending data with the length of 177 to the IoT platform.

- The SoftRadio verifies data validity after receiving the data and replies OK to the water meter after the verification succeeds.

- The SoftRadio sends an MO request to the IoT platform. The MO request contains data reported by the water meter. After receiving a response from the IoT platform, the SoftRadio sends multiple MT requests to the IoT platform. The IoT platform replies data to be delivered to the water meter to the SoftRadio. The SoftRadio caches the data.

- The water meter sends an AT+NMGR command to obtain downlink data stored in the SoftRadio cache. The SoftRadio replies the earliest data with the length of 23 to the water meter in +NMGR mode. The water meter sends an AT+NMGR command again. The SoftRadio replies data with the length of 21 to the water meter. 
The water meter sends an AT+NMGR command the third time. The SoftRadio does not reply to the water meter because there is no data in the SoftRadio cache.

- The water meter sends an AT+NMGS command again. The SoftRadio sends data with the length of 17 to the IoT platform.

- The SoftRadio confirms that the data is valid after receiving the request and replies $\mathrm{OK}$.

- The SoftRadio sends an MO request to send data to the IoT platform and then sends an MT request to the IoT platform to download data to be delivered to the water meter. [9]

\section{Conclusion}

This article provides an overview of NB-IoT \& eMTC as one of the main $5 \mathrm{G}$ three KPIs (massive IoT connections, low latency and high gigabit throughput) and discusses the RF deployment scenarios of $5 \mathrm{G}$ including RF KPI: coverage and capacity. Our trial results show that the targets can be achieved in all deployment scenarios of $5 \mathrm{G}$.

\section{Acknowledgement}

This paper is supported by Huawei and Ericsson, 5G/IoT Tech Lab, Maroc Telecom R\&D Center.

\section{$7 \quad$ References}

[1] LTE Evolution for IoT Connectivity, Nokia White Paper, 2016. https://www.openecosystem.org/assets/lte-evolution-iot-connectivity

[2] Ratasuk, R.; Prasad, A.; Zexian Li; Ghosh, A.; Uusitalo, M., "Recent advancements in M2M communications in 4G networks and evolution towards 5G," ICIN, Feb. 2015. 10.1109/ICIN.2015.7073806

[3] R1-157248, "NB IoT - Capacity evaluation," Nokia Networks, RAN1\#83, Anaheim, USA, 2015. https://portal.3gpp.org/ngppapp/CreateTdoc.aspx?mode=view\&contributionId $=670162$

[4] "Cellular networks for Massive IoT", Ericsson white paper, no. Uen 284 23-3278, published by Ericsson, January 2016. https://www.ericsson.com/assets/local/publications/whi te-papers/wp iot.pdf

[5] A. El Mahjoubi, T. Mazri, N. Hmina, "First Africa and Morocco NB-IoT experimental results and deployment scenario: new approach to improve main 5G KPIs for smart water management" 10.1145/3175628.3175641

[6] A. El Mahjoubi, T. Mazri, N. Hmina, "M2M and eMTC communications via NB-IoT Morocco first testbed experimental results and RF deployment scenario: New approach to improve main 5G KPIs and performances", 10.1109/WINCOM.2017.8238156

[7] Wanghui Li, Ganghua Bai, "Internet of Things System Based on Mobile Communication Network" https://doi.org/10.3991/ijoe.v14i11.9513 
Paper-NB-IoT and eMTC: Engineering results towards 5G/IoT Mobile Technologies

[8] Andreja Rojko "Industry 4.0 Concept: Background and Overview" https://doi.org/10.3991/ijim.v11i5.7072

[9] A. El Mahjoubi, T. Mazri, N. Hmina "4.5G Capacity \& QoS Optimization: A New Proposal to Enhance the Principal KPIs of 5G/IoT" https://doi.org/10.3991/ijoe.v14i11.9256

\section{Authors}

Dr. Ahmed El Mahjoubi received state engineer degree in networks \& telecommunication systems from the National School of Applied Sciences Kenitra, Morocco, in 2014. Since 2014 he has been a Technical Director in the Department of Network Performances Service (NPS), Huawei Technologies. His main research interests include 3G/4G/5G systems QoS/QoE/KPIs, Network Audit, Network Planning and Optimization, wireless communication systems especially in the topics of RF systems.

He has been involved in several national and international MBB projects on RF Systems: IAM 2G/3G SingleRAN Project, IAM LTE \& VoLTE Project, IAM GUL Project, INWI 3G/4G Project, INWI UMTS900 Project and Bouygues Telecom $4 \mathrm{G} / 5 \mathrm{G}$ Project. He has been the radio coordinator of the first test of $4 \mathrm{G}+$ in Morocco $\&$ Africa.

Prof. Tomader Mazri, HDR degree in Networks and Telecommunication from IbnTofail University, Ph.D. degree in Microelectronics and Telecommunication from SidiMohamed BenAbdellah University and INPT of Rabat, Master's degree in Microelectronics and Telecommunication Systems, Bachelor's degree in telecommunication from CadiAyyad university. She is currently a professor at National School of Applied Sciences of Kenitra and a Permanent member of Electrical and Telecommunications Engineering Laboratory. Author and co-author of twenty articles journals, forty articles in international conferences, a chapter and three books. Her major research interests are on Microwave systems for mobile and radar, Smart antennas and NG Mobile network.

Prof Nabil Hmina, Professor of Higher Education Director of the National School of Applied Sciences Kenitra, since November 2011 to date, Degree in Physics, Option: Thermodynamics - Mohammed V University, University PhD - Engineering Sciences, University and Ecole Centrale de Nantes, 1994,HDR (1st in Morocco) Ibn Tofail University, Kenitra, 2002. Vice-President for Academic Affairs and Information Technology of the University Ibn To U fail, 2005-2011 Post-Doctoral Researcher: EDF - thermokinetics Laboratory of Nantes, 1994-1995, Research Engineer at PolyTech school of Nantes, 1995-1997. Director of the research laboratory "Systems Engineering" since 2012 (15 permanent teachers 10 teachers Researchers Associate researchers $75 \mathrm{PhD}$ students. Author and / or co-author of a dozen articles in notorious newspapers and forty papers in international conferences. Jury President, member of several theses and Habilitations for research orientation.

Article submitted 16 October 2018. Resubmitted 17 November 2018. Final acceptance 20 November 2018. Final version published as submitted by the authors. 